among adult women of reproductive age and evaluate the appropriateness of empirical treatment.

Methods. Non-pregnant women between the age of 20 and 49 years who presented with vaginal discharge were prospectively enrolled in a teaching hospital since Oct 2018. Vaginal swabs were collected for determination of Nugent score, culture for Trichomonas vaginalis (TV) and Candida species, and multiplex polymerase chain reaction (PCR) for BV, VVC and TV. Demographics, symptoms, physical findings, and the empirical treatment were recorded.

Results. From Oct 2018 to May 2020, 172 women were included (median age, 37 years). The prevalence of laboratory confirmed BV, VVC, and TV was $21.5 \%$ $(\mathrm{n}=37), 20.3 \%$ (35), and $0.6 \%$ (1), respectively. Ten (5.8\%) women had concurrent BV and VCC. Among 38 women who had bacterial vaginosis or trichomoniasis, only 8 (21.1\%) received metronidazole empirically while more than half $(11 / 19,57.9 \%)$ of women who received metronidazole empirically did not have laboratory-confirmed bacterial vaginosis or trichomoniasis. Among 35 women who had candidiasis, 10 (28.6\%) received antifungal agents. Antifungal agents were prescribed to more than two thirds $(21 / 31,67.7 \%)$ of women who did not have laboratory confirmed candidiasis. Overall, 58.7\% (101/172) of empirical treatment was deemed optimal. Multiplex PCR test has an overall diagnostic accuracy of $86.0 \%$ (148/172) as compared to the composite gold standard.

Conclusion. The empirical treatment for vaginal discharge syndrome is suboptimal. Better diagnostic assays have a potential to improve clinical patient care.

Disclosures. All Authors: No reported disclosures

\section{Disruption of the Body Temperature Circadian Rhythm} in Hospitalized Patients

Ivayla I. Geneva, MD, $\mathrm{PhD}^{1}$; Waleed Javaid, MD, FACP, FIDSA, FSHEA ${ }^{2}$; ${ }^{1}$ State

University of New York Upstate, Syracuse, New York ${ }^{2}$ Mount Sinai Health System, Brooklyn, NY

Session: P-55. New Approaches to Diagnostics

Background. The circadian rhythm is believed to offer survival advantage with dysregulation being linked to immune response deficiencies and metabolic derangements. Diurnal temperature variation exists in humans, yet its preservation during illness is not well understood. Herein we present an analysis of diurnal body temperatures among hospitalized patients, with a focus on infectious versus non-infectious diagnoses.

Methods. Temperatures measured within $1 / 2$ hour of $8 \mathrm{am}, 12 \mathrm{pm}, 4 \mathrm{pm}, 8 \mathrm{pm}, 12$ am, and 4 am from 16,245 hospitalized patients were analyzed using descriptive statistics and t-tests.

Results. Although we found a diurnal pattern when analyzing the ensemble of temperatures from all patients (Figure 1), stratified by measurement site (oral, axillary, temporal, and tympanic), the through-to-peak difference was only $0.2 \mathrm{~F}(0.1 \mathrm{C})$, while previously reported diurnal difference in healthy volunteers was $1.9^{\circ} \mathrm{F}\left(1.06^{\circ} \mathrm{C}\right)$. Data from the core body temperature sites monotherm and rectal did not show any diurnal pattern. The peaks in body temperature occurred at $8 \mathrm{pm}$ for all patients, regardless of age, which is similar to healthy people. However, the minimum body temperature was shifted to later times compared with healthy people (6am or 2 hours before rising in health) - for young patients (age 20-30 years, $\mathrm{N}=1285$ ) the through was at $8 \mathrm{am}$ and for elderly patients (age $70-80$ years, $\mathrm{N}=1736$ ), it was at $12 \mathrm{pm}$ (Figure 2). Analysis of body temperature of individual patients showed that less than $20 \%$ of patients exhibited diurnal variation and among those showing variation, the trend was present only on the minority of hospitalization days (Table 1). Interestingly, the presence or absence of an infectious process did not influence the proportion of patients showing diurnal variation. Figure 1

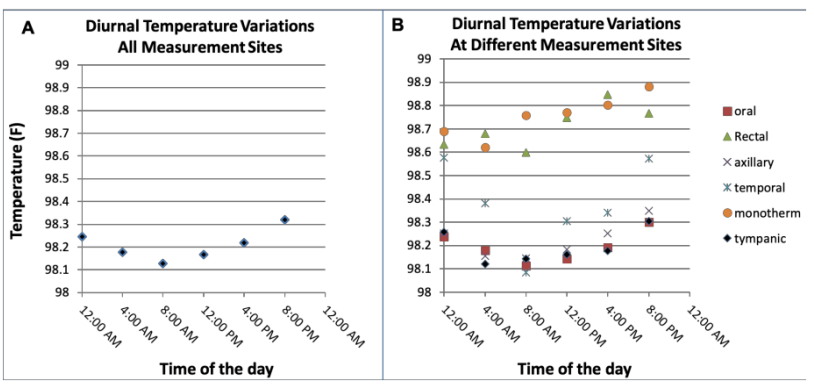

Figure 2

\section{Diurnal Temperature Variation and Age}

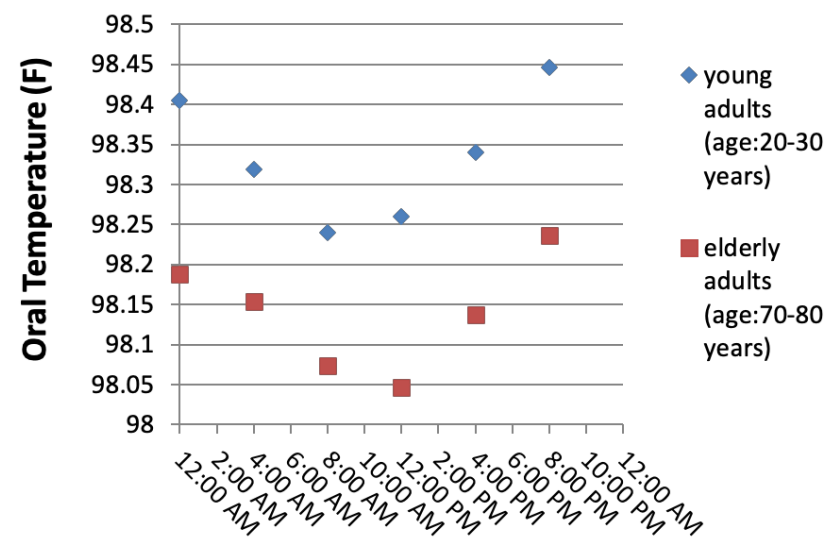

Time of the day

Table 1

\begin{tabular}{|r|c|c|c|c|}
\hline & $\begin{array}{c}\text { diurnal trend } \\
\text { present }\end{array}$ & $\begin{array}{c}\text { no diurnal } \\
\text { trend }\end{array}$ & $\begin{array}{c}\% \text { with } \\
\text { diurnal trend }\end{array}$ \\
\hline no infection & ACS rule out & 1 & 6 & 14 \\
\hline AAA & 0 & 2 & 0 \\
\hline infection present & suicidal ideation & 1 & 1 & 50 \\
\hline & abdominal abscess & 1 & 4 & 20 \\
\hline & pneumonia & 0 & 3 & 0 \\
\hline total infection present & 1 & 7 & 13 \\
\hline
\end{tabular}

Conclusion. Hospitalization is associated with disruption in the circadian rhythm as reflected by patients' body temperature, with shifting of the diurnal variation curve and blunting of the temperature range both in the ensemble and on the individual level. The trend is not influenced by having an infection. However, since core body temperatures tend to be the measurement site of choice in the ICU setting, we suspect that further obliteration of the diurnal rhythm occurs with more severe disease.

Disclosures. All Authors: No reported disclosures

\section{Evaluation of the FilmArray ${ }^{\oplus}$ Global Fever Panel}

Jared R. Helm, $\mathrm{PhD}^{1}$; Brian Jones, $\mathrm{PhD}^{2}$; Corike Toxopeus, $\mathrm{PhD}^{2}$; David S. Rabiger, $\mathrm{PhD}^{3}$; Mark Gurling, $\mathrm{PhD}^{2}$; Madeline Veloz, n/a $\mathrm{a}^{3}$; Alex J. Kelley, n/a ${ }^{2}$; Olivia Jackson, $\mathrm{n} / \mathrm{a}^{3}$; Marissa Burton, BS Biology ${ }^{4}$; Sidney Maxey, Bachelor's of Science in Biology ${ }^{3}$; Natalie Batty, $\mathrm{MPH}^{3}$; Ashley Wiltsie, BS in Biology ${ }^{2}$; Haley Halberg, BS Molecular Cell Biology ${ }^{2}$; Pascal Belgique, $\mathrm{BS}^{3}$; Cynthia Andjelic, $\mathrm{PhD}^{2}$; Cynthia L. Phillips, $\mathrm{PhD}^{3} ;{ }^{1}$ Biofire Defense LLC, Riverton, Utah; ${ }^{2}$ BioFire Defense, LLC, Salt Lake City, Utah ${ }^{3}$ BioFire Defense, Salt Lake City, Utah ${ }^{4}$ Biofire Defense, LLC, Sandy, Utah

Session: P-55. New Approaches to Diagnostics

Background. Acute Febrile Illness (AFI) is caused by a diverse set of pathogens. The FilmArray Global Fever (GF) Panel, developed by BioFire Defense in collaboration with the U.S. Department of Defense and NIAID, uses an automated, multiplex nested PCR system to evaluate whole blood samples for multiple pathogens simultaneously in under an hour.

Methods. BioFire Defense conducted analytical performance studies to show sensitivity (LoD), inclusivity, and specificity (exclusivity), and a prospective clinical study to evaluate the positive percent agreement (PPA) and negative percent agreement (NPA) of the GF Panel. The results of these studies will be reported in two submissions to the US FDA. 